\title{
Nodal signalling and apoptosis
}

\author{
Hongmei Wang ${ }^{1,2}$ and Benjamin K Tsang ${ }^{2}$ \\ ${ }^{1}$ State Key Laboratory of Reproductive Biology, Institute of Zoology, Chinese Academy of Sciences, Beijing 100080, \\ China and ${ }^{2}$ Departments of Obstetrics and Gynaecology and Cellular and Molecular Medicine, Ottawa Health \\ Research Institute, University of Ottawa, Ottawa, Ontario, K1Y 4E9, Canada
}

Correspondence should be addressed to H Wang; Email: wanghm@ioz.ac.cn

B K Tsang; Email: btsang@ohri.ca

\begin{abstract}
Nodal, a member of the transforming growth factor $\beta$ family, was first cloned from a 7.5 day post-coitum mouse embryo cDNA library. Nodal exerts its biological effects by signalling through its types I and II serine/threonine kinase receptor complex and intracellular Smad proteins. The type II receptors for Nodal are Activin type II receptors ActRIIA and ActRIIB, whereas the putative type I receptors are Activin receptor like kinase (ALK) 4 and ALK7. The main Smad proteins involved in Nodal signalling are Smad2 and Smad3. Studies of Nodal in adult tissues indicate that it is pro-apoptotic in rat ovarian granulosa cells, human trophoblast cells and human ovarian epithelial cancer cells and is growth inhibitory in the latter two cell types. This review summarises the progress made on the functions of Nodal in the apoptosis of adult tissues, especially in the ovary and placenta.
\end{abstract}

Reproduction (2007) 133 847-853

\section{Introduction}

Apoptosis is an evolutionarily conserved mode of programmed cell death across the animal kingdom. It plays important roles in numerous physiological events in mammals, including ovarian follicular atresia, decidualisation and placentation during embryo implantation, as well as many pathological conditions. Nodal, a member of the transforming growth factor (TGF) $\beta$ family, exerts its biological effects by signalling through a cell surface serine/threonine kinase receptor complex of types II and I receptors and intracellular Smad proteins. Nodal plays an important role in the induction of dorsal mesoderm, anterior patterning and formation of leftright asymmetry during early embryo development. In extra-embryonic tissues, studies have been limited. Nodal is capable of inducing apoptosis and inhibiting cell growth. This review will focus on the role of Nodal in apoptosis in the ovary and placenta, as well as its possible physiological and pathophysiological roles in folliculogenesis, follicular atresia, ovarian cancers and placentation.

\section{TGF $\beta$ family members and their signalling mechanisms}

The transforming growth factor (TGF) $\beta$ family consists of a large group of growth and differentiation factors, including several TGF $\beta$ proteins, bone morphogenetic proteins (BMPs), Activins/Inhibins, growth and differentiation factors (GDFs), as well as Nodal and its related proteins (Massague \& Chen 2000, Chang et al. 2002). Members of TGF $\beta$ family are multifunctional proteins that regulate cell proliferation and differentiation, extracellular matrix modification, angiogenesis, apoptosis and immunosuppression (Schier \& Shen 2000, Feng \& Derynck 2005, Jones et al. 2006).

A TGF $\beta$ protein exerts its function by binding to and bringing together on the cell surface types I and II receptors to form a ternary ligand-receptor complex (Massague 1998). Five members of type II and seven members of type I receptors (Activin receptor like kinase (ALK) 1-7) have been characterised in mammals (Peng 2003). Upon phosphorylation by the type II receptor, the type I receptor phosphorylates and activates Smads, which are intracellular signalling molecules for members of the TGF $\beta$ superfamily. Smad2 and Smad3 respond to TGF $\beta$ s, Activins, Nodal and Lefty, whereas Smad1, Smad5 and Smad8 mediate BMP signalling (Miyazawa et al. 2002). Following receptor activation, phosphorylated Smads are released from the receptors and form oligomeric complexes with common-partner Smad, Smad4, and translocate into the nucleus to regulate the transcription of target genes. 


\section{Nodal}

Nodal was first cloned from a 7.5 day post-coitum mouse embryo cDNA library (Zhou et al. 1993). Like other members of the TGF $\beta$ family, Nodal exerts its biological effects by signalling through its type I and type II serine/ threonine kinase receptor complex and Smad2, Smad3 and Smad4. The receptor-activated Smad2 and Smad3 form a complex with Smad4, which is imported into the nucleus where they, with the aid of transcriptional activators or inhibitors, bind to the cis-acting elements in the promoter region of the target gene to modulate gene transcription (Findlay et al. 2001).

ALK4 and ALK7 are the two putative type I receptors for Nodal (Tsuchida et al. 1996, Reissmann et al. 2001). The type II receptors for Nodal are thought to be Activin type II receptors ActRIIA and ActRIIB. ALK7 was first cloned from the rat as an orphan receptor (Tsuchida et al. 1996). The serine/threonine kinase domain of ALK7 is similar to that of ALK4 but its extracellular domain is unique and bears no similarity to that of any other ALKs. These features suggest that ALK7 may possess signalling properties similar to those of other TGF $\beta$ and Activin receptors but have different ligand specificity. Indeed, ALK7 is not capable of binding TGF $\beta$, Activin or BMP7 even in the presence of the type II receptors as reported by Reissmann et al. (2001). However, Activin AB and Activin $B$ are thought to be ligands for ALK7 in the pancreatic $\beta$ cell line MIN6 (Tsuchida et al. 2004). Recent study with gonadotrope cells also revealed that Activin B signals through both ALK4 and ALK7 (Bernard et al. 2006).

Based on studies in the mouse, Xenopus and zebrafish (Zhang et al. 2004), Nodal is identified as a critical regulator of early vertebrate development which is involved in the induction of dorsal mesoderm, anterior patterning and formation of left-right asymmetry (lannaccone et al. 1992, Brennan et al. 2002, Eimon \& Harland 2002). Nodal knockout is lethal, resulting from defects in primitive streak formation (Gu et al. 1998). On the other hand, Nodal inhibits the differentiation of rat trophoblast stem cells into giant cells (lannaccone et al. 1992, Ma et al. 2001). Similarly, it has been suggested that Nodal regulates human placental development (Roberts et al. 2003). Pro-apoptotic and growth inhibitory effects of Nodal have been reported in ovarian granulosa cells (Wang et al. 2006), human trophoblast cells (Munir et al. 2004) and ovarian epithelial cancer cells (Xu et al. 2004, 2006). Moreover, activation of ALK7 is capable of inducing apoptosis in hepatoma cells (Kim et al. 2004) and pancreatic $\beta$ cells (Zhang et al. 2006).

\section{Nodal and ovarian follicular atresia}

In the ovary, more than $99 \%$ of the developing follicles fail to mature fully but instead undergo atresia
(Amsterdam et al. 1997). Follicular atresia is a negative and selective degenerative process during follicular growth and development, and occurs as a consequence of apoptotic cell death of the follicular somatic cells and of the oocyte (Chun \& Hsueh 1998, Asselin et al. 2001). The destiny of the developing follicles (continual growth and eventual ovulation or atresia) is dependent on the fate of the granulosa cells within them (i.e. proliferation, differentiation or apoptosis), which is determined by the coordinated actions and interactions of cell survival and death factors, including follicle-stimulating hormone (FSH), GDF-9, Nodal and other growth factors and cytokines (Jiang et al. 2003, Craig et al. 2007). In mammals, two basic apoptotic pathways have been defined: the mitochondria-mediated pathway and the death receptor pathway (Hussein et al. 2003). Interaction between pro- and anti-apoptotic members of the $\mathrm{Bcl}-2$ family of proteins in the mitochondrion determines whether pathways for apoptosis are activated or suppressed (Zinkel et al. 2006). Release of mitochondrial cytochrome $C$, second mitochondria-derived activator of caspase (Smac/DIABLO) and Omi/HtrA2 activates apoptosis (Labedzka et al. 2006). Cytochrome $c$ forms a complex (apoptosome) with apoptosis-activating factor-1 (Apaf1) and procaspase-9, thus activating caspase-9. Activated caspase-9 cleaves and activates downstream effector caspases, including caspase-3, resulting in induction of apoptosis (Hussein 2005).

Nodal and its type I receptor ALK7 are expressed in a cell type-specific and follicular stage-dependent manner during folliculogenesis (Wang et al. 2006). Nodal immunoreactivity in granulosa cells is the strongest in pre-antral follicles when compared with the signals from later stages of development. In addition, immunosignals of Nodal are also detected in the theca cell layer of antral follicles. In contrast, ALK7 was mainly detected in the interstitial cells at the pre-antral stage of development, while it was present in granulosa cells, but not theca cells in antral follicles. Throughout antral growth, ALK7 is highly expressed in granulosa cells, whereas Nodal content in the granulosa cells decreases as the follicle transits from the pre-antral to antral stage of development, implying that during development through the penultimate stage, the granulosa cell acquires their ability to undergo apoptosis and initiate atresia (due to the presence of ALK7) but fail to do so due to low level of its ligand (Wang et al. 2006). During declining gonadotrophin support (e.g. artificial gonadotrophin withdrawal; Boone et al. 1997), the antral follicle destined for atresia not only expresses an increased level of Nodal but also shows co-localisation of both the ligand and its receptor in the granulosa cells. In addition, the relative abundance of Nodal mRNA is significantly higher in granulosa cells from atretic antral follicles than healthy ones, suggesting that increased granulosa cell Nodal expression may be a physiological signal for the induction of atresia. 
Addition of recombinant Nodal to cultures of granulosa cells from large antral and pre-ovulatory follicles significantly increases granulosa cell apoptosis (Wang et al. 2006). Increased apoptosis can also be observed in granulosa cells infected with adenoviral Nodal when compared with LacZ control (Wang et al. 2006), suggesting that Nodal is a death signal for granulosa cells. Granulosa cells co-infected with adenoviral-dominant negative ALK7 and Nodal exhibit lower extent of apoptosis when compared with the cells infected with Nodal alone, providing support to the concept that Nodal is able to activate endogenous ALK7 (Wang et al. 2006). In addition, forced expression of an activated ALK7 mimics the pro-apoptotic effect of Nodal and significantly increases granulosa cell apoptosis, thus providing support that the Nodal/ALK7-signalling pathway is pro-apoptotic in granulosa cells (Wang et al. 2006).

It has been demonstrated that Nodal binds to ALK7 or ALK4 and activates the Smad2/3 signalling pathway (Findlay et al. 2001). Granulosa cells infected with adenoviral Nodal or an activated ALK7 exhibit increased phospho-Smad2 (ser465/467) level and phosphoSmad2/Smad2 ratio, and increased nuclear import of phopsho-Smad2 and apoptosis in the granulosa cells, suggesting a role of Smad2 in Nodal/ALK7-mediated granulosa cell apoptosis (Wang et al. 2006).

It has been demonstrated that Nodal or ALK7 activation down-regulates granulosa cell X-linked inhibitor of apoptosis protein (XIAP) mRNA and protein content (Wang et al.). XIAP, a well studied member of the inhibitor of apoptosis protein family, is a determinant of granulosa cell survival and follicular growth (Asselin et al. 2001). XIAP is an intracellular anti-apoptotic protein, which functions as a direct inhibitor of caspases3, -7 (Deveraux et al. 1997) and -9 (Deveraux et al. 1998). XIAP can also function as a $E 3$ ubiquitin ligase for caspase-3 (Yang et al. 2000, Suzuki et al. 2001) and Smac (Hu \& Yang 2003). In addition, XIAP can bind to Smac/DIABLO and HtrA2/Omi, leading to the neutralisation of death signals in the cytosol and preventing apoptosis (Datta et al. 1999, Chung et al. 2002). Furthermore, ALK7-mediated apoptosis can be attenuated by forced expression of XIAP or facilitated by XIAP down-regulation (Wang et al. 2006). Dominant negative Smad2 expression attenuates ALK7-mediated XIAP down-regulation, suggesting that the decrease in XIAP content by Nodal involves Smad signalling.

The phosphotidyl inositide-3 kinase (PI3K)/Akt pathway plays an important role in regulating granulosa cell apoptosis (Asselin et al. 2001). Akt, also known as protein kinase $(\mathrm{PK}) \mathrm{B}$, is a serine/threonine PK downstream of PI3K (Bellacosa et al. 1991, Cheng et al. 1992, Nakatani et al. 1999) and an important anti-apoptotic factor. A number of Akt substrates are pro-apoptotic, including Bcl-2-associated death promoter (BAD), caspase-9, forkhead transcription factors and apoptosis signal-regulating kinase 1 (ASK1) (Datta et al. 1999, Kim et al. 2001). Their function is attenuated by Akt phosphorylation. Overexpression of Nodal or ALK7 activation can significantly decrease phospho-Akt and phospho-Akt/Akt ratio. Furthermore, activation of Akt decreases ALK7-mediated mitochondrial Smac, Omi and cytochrome $c$ release and attenuates granulosa cell apoptosis (Wang et al. 2006).

Although the above studies have significantly advanced our current understanding on the molecular and cellular mechanisms regulating follicular development and atresia, much remains to be learned on the regulation on follicular Nodal and ALK7 expression during folliculogenesis and their downstream signalling pathways involved in the regulation of follicular cell fate and follicle destiny. Wang et al. (2006) proposed a hypothetical model to guide possible future investigations. In the healthy antral follicles, Nodal is localised in the theca cells, whereas its type I receptor ALK7 is in the granulosa cells. Uncoupling of the ligand from its receptor renders the Nodal-signalling pathway silent. FSH increases XIAP expression and activates the PI3K-Akt pathway. The up-regulation of Akt and XIAP suppresses the release of mitochondrial death proteins and therefore inhibits caspase activation and activities, and promotes cell survival (Wang et al. 2003). However, in mid- to late-follicular stages, FSH levels decline and induce the co-localisation of Nodal and ALK7 in granulosa cells, triggering ALK7 signalling and downstream events, including Smad2 activation, Akt inhibition and XIAP down-regulation. Inactivation of Akt increases mitochondrial release of Smac, Omi and cytochrome $c$, thus activating granulosa cell caspases and apoptosis, and eventually follicular atresia.

\section{Nodal and apoptosis in ovarian cancer cells}

Ovarian cancer is the most fatal gynaecological malignancy in the Western world and ranks fifth among the most common female cancers (Ries et al. 2000). Ovarian cancer that originates from the ovarian surface epithelium constitutes $\sim 90 \%$ of all cancers in the ovaries (Auersperg et al. 2001). The homeostasis of human ovarian surface epithelial cells is maintained by a delicate balance in the expression and actions of tumour suppressors and cell survival factors (Cheng et al. 2002). Dysregulation of the homeostatic mechanism(s) may lead to ovarian cancers and to chemoresistance. mRNAs for Nodal, ActRIIB, the type II receptor partner of ALK7 and Smad2, 3 and 4, as well as four transcripts of ALK7 (ALK7-1, -2, -3 and -4) are expressed in both chemosensitive and chemoresistant ovarian cancer cell lines (Xu et al. 2004). Overexpression of Nodal significantly increases apoptosis, a response that could be mimicked by introduction of the constitutively active form of ALK7 (ALK7-ca; Xu et al. 2004). Interestingly, ALK7-ca and Nodal induce a greater extent of apoptosis in the 
chemosensitive cells (A2780-s) when compared with its chemoresistant counterpart (A2780-cp), which is associated with decreased XIAP expression at both mRNA and protein levels (Xu et al. 2004). XIAP levels in Nodaltransfected or ALK7-activated chemosensitive cells are also significantly lower than those in the chemoresistant variant, implying that the responsiveness of the ovarian cancer cells to Nodal and ALK7 activation may be related to the cellular level of XIAP (Xu et al. 2004). This is also consistent with the observation that activation of the Nodal/ALK7 pathway also significantly increases the cleavage/activation of caspases-9 and -3 (Xu et al. 2004). Since XIAP is an inhibitor of caspases-9 and -3, downregulation of XIAP by Nodal may be the mechanism by which Nodal activates caspases-3 and -9 and induces apoptosis in ovarian cancer cells (Xu et al. 2004).

Using the immortalised ovarian surface epithelial cell line IOSE397 and the ovarian cancer cell line OV2008 $\mathrm{Xu}$ et al. (2006) found that both normal and malignant ovarian epithelial cell lines expressed the signalling molecules of the Nodal-ALK7 pathway, including the ligand, receptors and Smads. Activation of ALK7 effectively induces apoptosis, which is accompanied by up-regulation of pro-apoptotic Bax and down-regulation of anti-apoptotic BCl-2 and $\mathrm{BCl}-\mathrm{XL}$, disruption of the mitochondrial membrane potential as well as increased mitochondrial release of cytochrome $c$ (Xu et al. 2006). It has been proposed that Nodal activates ActRIIB and ALK7 and induces $S m a d 2 / 3$ activation, which in turn regulates the expression of $\mathrm{Bax}$ and $\mathrm{Bcl}-2$. The decrease in $\mathrm{Bcl}-2 /$ Bax ratio results in the release of cytochrome $c$, activation of caspase-3 and subsequent apoptosis (Xu et al. 2006).

Our knowledge on the mechanism(s) by which Nodal/ALK7 signalling induces apoptosis remains incomplete. Overexpression of an activated ALK7 can also induce apoptosis in hepatoma cells and pancreatic $\beta$ cells (Kim et al. 2004, Zhang et al. 2006). However, since Activin $A B$ and Activin $B$ can also bind to ALK7 (Tsuchida et al. 2004, Bernard et al. 2006), the ability of ALK7 to induce apoptosis does not in itself implicate Nodal as a pro-apoptotic ligand. However, several mechanisms of ALK7-induced apoptosis have been proposed, although available evidence is limited (Kim et al. 2004, Zhang et al. 2006), including: (1) ALK7mediated c-Jun $\mathrm{N}$-terminal kinase (JNK) activation. It has been demonstrated that ALK7 activation increases levels of active and phosphorylated JNK, but not of p38 or extracellular signal-regulated kinase (Erk). Expression of dominant negative form of stress-activated PK/Erk kinase (SEK), an upstream kinase of JNK, abolishes ALK7induced JNK activation and inhibits apoptosis (Kishimoto et al. 2003). (2) Activation of mitochondria- but not death receptor-mediated cell death pathway. This notion is supported by the observation that ALK7-induced apoptosis in $\mathrm{FaO}$ rat hepatoma cells is accompanied by activation of caspases- 3 and -9 , but not by caspases-7 and -8. In addition, the pan-caspase inhibitor Z-VAD- fmk significantly attenuates ALK7-induced apoptosis which further support the conclusion that caspase activation is necessary for the induction of apoptosis. On the other hand, ALK7 induces SEK1-mediated mitochondrial cytochrome $c$ release, which is necessary for the formation of the apoptosome with Apaf1 and procaspase-9 (Greenleaf et al. 1979). (3) Activation of Smad3 pathway. Overexpression of ALK7 in $\mathrm{FaO}$ rat hepatoma and Hep3B human hepatoma cells activates Smad2 and Smad3 (Kim et al. 2004). Smad3 RNA interference in the ALK7-infected Hep3B cells significantly inhibits caspase-3 cleavage (Kim et al. 2004), suggesting that Smad3 is indispensable in ALK7-induced apoptosis. (4) Suppression of Akt activation and the activation of the Smad2-caspase-3 cascade. This notion is supported by the observation that ALK7 activation in pancreatic $\beta$ cells increases Smad2 phosphorylation, reduces Akt kinase activity and increases levels of active caspase-3 (Zhang et al. 2006).

\section{Nodal and apoptosis during plancentation}

During early placentation, the human trophoblast differentiates into the villous and extravillous trophoblasts (Huppertz et al. 2006). In both types of trophoblast, apoptosis plays a role in normal cell turnover, ensuring that non-functional cells are eliminated without a local inflammatory reaction from the maternal host (Huppertz et al. 2006). Nodal mRNA is expressed in the placenta at various gestational ages and in the choriocarcinoma cell line JEG-3 cells. mRNAs for ALK7 are also present in the human placentae, with all four transcripts of ALK7 present also in JEG-3 cells (Xu et al. 2004). Placental ALK7 from all three trimesters of pregnancy migrates on SDS-PAGE as two bands of 58 and $52 \mathrm{kDa}$, probably attributable to the glycosylated forms of the full-length and truncated ALK7 (tALK7) respectively. Full-length ALK7 content is constant throughout gestation, whereas tALK7 exhibits a significantly higher level in the third trimester than in the earlier stages. Overexpression of ALK7 and Nodal markedly increases apoptosis in JEG-3 cells (Munir et al. 2004). In addition, an increased level of active caspase-3 is detected in cells transfected with Nodaland ALK7-Ca, implying a role of Nodal in the induction of apoptosis during normal placentation (Munir et al. 2004). The physiological role of the Nodal/ALK7 pathway during pregnancy is not known, nor is its possible involvement in the aetiology of pregnancy complications such as preeclampsia, intrauterine growth restriction and habitual abortion.

\section{Future studies}

This review focuses on the function of Nodal in apoptosis in the ovaries and placenta. Apoptosis occurs in the mammalian ovaries under physiological conditions and 


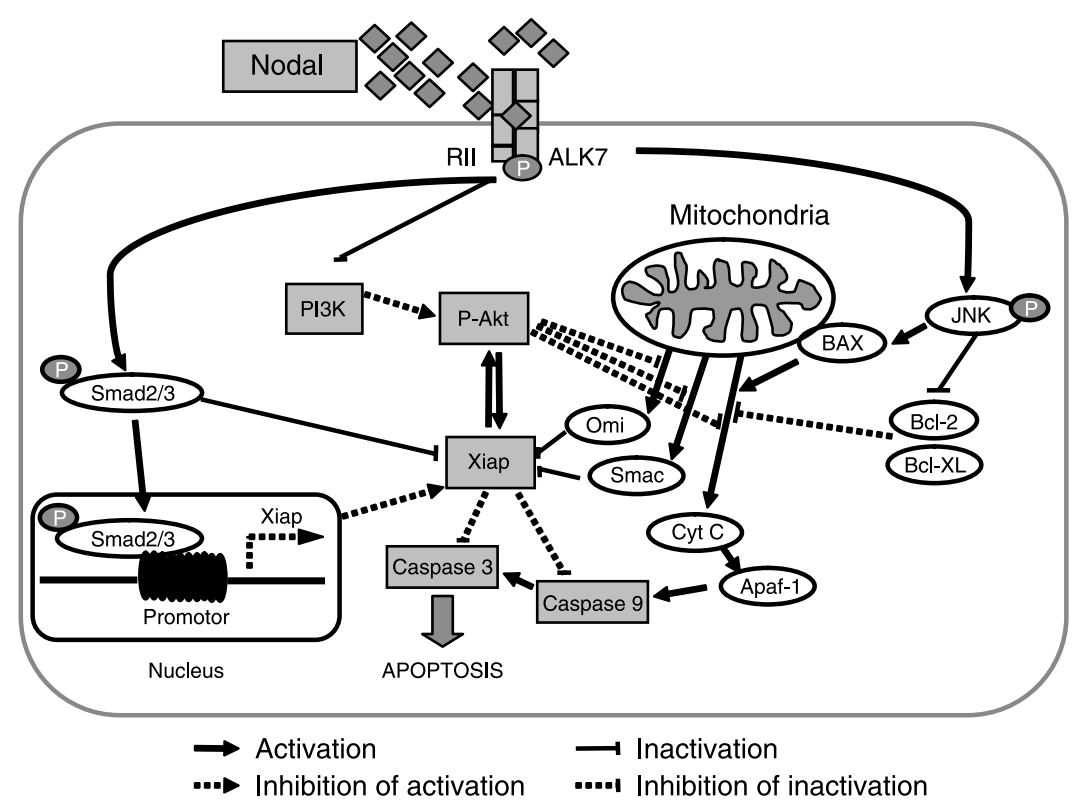

Figure 1 Summarised apoptotic pathways triggered by Nodal/ALK7 signalling. Nodal induces apoptosis via activation of its type I receptor, ALK7, which (1) stimulates the phosphorylation of Smad2 and Smad3, resulting in the down-regulation of XIAP, (2) activates JNK signalling which downregulates $\mathrm{Bcl}-2$ and $\mathrm{BCl}-\mathrm{X}$ and up-regulates Bax, (3) inhibits Akt and triggers the mitochondrial release of Smac, Omi and Cytochrome $c$. These responses in turn activate caspases and ultimately induce apoptosis.

plays important roles in ovarian folliculogenesis and follicular atresia. During embryo implantation, apoptosis is important for decidualisation and placentation (Huppertz et al. 2006). Dysregulation in apoptosis leads to pathological states, including cancers, pregnancy-related diseases or ovarian dysfunctions. Several recent studies have indicated that Nodal is able to induce apoptosis in ovarian granulosa cells, ovarian epithelial cancer cells and choriocarcinoma cells. Furthermore, these studies have established that Nodal and ALK7 activation down-regulates the cell survival factors XIAP and Akt, activates the mitochondrial death pathway as well as caspases-3 and -9 , but not caspases- 7 and -8 . The signalling mechanism of Nodal-induced apoptosis is summarised in Fig. 1. However, much remains to be learned about the precise molecular mechanism of Nodal-induced apoptosis in these reproductive tissues and the physiological relevance of these findings in other reproductive events.

Nodal exerts its biological function via type I receptors ALK4 and ALK7 and intracellular Smad2/3. The above studies only shed light on the importance of ALK7, but not ALK4, in Nodal-induced apoptosis. Whether ALK4 is involved in Nodal-induced apoptosis has not been reported. In addition, Smad3 is only known to be necessary for ALK7-induced apoptosis in hepatoma cells. However, Smad2 is indispensable in Nodal/ALK7induced apoptosis based on the results from ovarian granulosa cells and cancer cells, as well as from placental tissues. Whether indeed both Smad2 and Smad3 are equally important in Nodal-induced apoptosis and whether their involvement is cell type-dependent need to be further investigated. Equal attention should also be drawn to study the molecular mechanisms involved in Nodal-induced apoptosis.

Combinatorial interactions of heteromeric TGF $\beta$ receptors and Smad complexes provide a high degree of signalling specificity and versatility. While Nodal, Activin $A B$ and Activin $B$ share the same type I receptor ALK7, TGF $\beta$ s, Nodal, Activin, GDF-9 and Lefty activate the same R-Smads, suggesting that crosstalks between Nodal and other members of TGF $\beta$ family may be operational. Activation of Smads by different ligands may result in different cellular response. For example, although both GDF-9 and Nodal activate Smad2, the former promotes folliculogenesis while the later induces follicular atresia. This suggests that, in addition to the activation of Smads, other signalling pathways may also be involved in the action of these TGF $\beta$ family members. Their identity and the nature of the interactions with the Smads signalling pathway also need to be further investigated.

\section{Acknowledgements}

This work was supported by grants from the Canadian Institutes of Health Research (CIHR; MOP 10369 to BKT) and '973 Program' from the Ministry of Science and Technology of the People's Republic of China (MOST; 2006CB504006 and 2006 CB944008 to HMW). HMW is a recipient of a Fellowship Award of the Strategic Training Initiatives in the Research in Reproductive Sciences funded by the Canadian Institutes of Health Research. The authors declare that there is no conflict of interest that would prejudice the impartiality of this scientific work. 


\section{References}

Amsterdam A, Dantes A, Selvaraj N \& Aharoni D 1997 Apoptosis in steroidogenic cells: structure-function analysis. Steroids $\mathbf{6 2}$ 207-211.

Asselin E, Wang Y \& Tsang BK 2001 X-linked inhibitor of apoptosis protein activates the phosphatidylinositol 3-kinase/Akt pathway in rat granulosa cells during follicular development. Endocrinology 142 2451-2457.

Auersperg N, Wong AS, Choi KC, Kang SK \& Leung PC 2001 Ovarian surface epithelium: biology, endocrinology, and pathology. Endocrine Reviews 22 255-288.

Bellacosa A, Testa JR, Staal SP \& Tsichlis PN 1991 A retroviral oncogene, akt, encoding a serine-threonine kinase containing an SH2-like region. Science 254 274-277.

Bernard DJ, Lee KB \& Santos MM 2006 Activin B can signal through both ALK4 and ALK7 in gonadotrope cells. Reproductive Biology and Endocrinology 452.

Boone DL, Carnegie JA, Rippstein PU \& Tsang BK 1997 Induction of apoptosis in equine chorionic gonadotropin (eCG)-primed rat ovaries by anti-eCG antibody. Biology of Reproduction 57 420-427.

Brennan J, Norris DP \& Robertson EJ 2002 Nodal activity in the node governs left-right asymmetry. Genes and Development $\mathbf{1 6}$ 2339-2344.

Chang H, Brown CW \& Matzuk MM 2002 Genetic analysis of the mammalian transforming growth factor-beta superfamily. Endocrine Reviews 23 787-823.

Cheng JQ, Godwin AK, Bellacosa A, Taguchi T, Franke TF, Hamilton TC, Tsichlis PN \& Testa JR 1992 AKT2, a putative oncogene encoding a member of a subfamily of protein-serine/threonine kinases, is amplified in human ovarian carcinomas. PNAS 89 9267-9271.

Cheng JQ, Jiang X, Fraser M, Li M, Dan HC, Sun M \& Tsang BK 2002 Role of $\mathrm{X}$-linked inhibitor of apoptosis protein in chemoresistance in ovarian cancer: possible involvement of the phosphoinositide-3 kinase/Akt pathway. Drug Resistance Updates 5 131-146.

Chun SY \& Hsueh AJ 1998 Paracrine mechanisms of ovarian follicle apoptosis. Journal of Reproductive Immunology 39 63-75.

Chung J, Bachelder RE, Lipscomb EA, Shaw LM \& Mercurio AM 2002 Integrin (alpha 6 beta 4) regulation of elF-4E activity and VEGF translation: a survival mechanism for carcinoma cells. Journal of Cell Biology 158 165-174.

Craig J, Orisaka M, Wang HM, Orisaka S, Thompson W, Zhu C, Kotsuji F \& Tsang BK 2007 Gonadotropin and intra-ovarian signals regulating follicle development and atresia: the delicate balance between life and death. Frontiers in Bioscience 12 3628-3639.

Datta SR, Brunet A \& Greenberg ME 1999 Cellular survival: a play in three Akts. Genes and Development 13 2905-2927.

Deveraux QL, Takahashi R, Salvesen GS \& Reed JC 1997 X-linked IAP is a direct inhibitor of cell-death proteases. Nature 388 300-304.

Deveraux QL, Roy N, Stennicke HR, Van Arsdale T, Zhou Q, Srinivasula SM, Alnemri ES, Salvesen GS \& Reed JC 1998 IAPs block apoptotic events induced by caspase- 8 and cytochrome $c$ by direct inhibition of distinct caspases. EMBO Journal 17 2215-2223.

Eimon PM \& Harland RM 2002 Effects of heterodimerization and proteolytic processing on Derriere and Nodal activity: implications for mesoderm induction in Xenopus. Development 129 3089-3103.

Feng XH \& Derynck R 2005 Specificity and versatility in tgf-beta signaling through Smads. Annual Review of Cell and Developmental Biology 21 659-693.

Findlay JK, Drummond AE, Dyson M, Baillie AJ, Robertson DM \& Ethier JF 2001 Production and actions of inhibin and activin during folliculogenesis in the rat. Molecular and Cellular Endocrinology $180139-144$.

Greenleaf AL, Borsett LM, Jiamachello PF \& Coulter DE 1979 Alphaamanitin-resistant $D$. melanogaster with an altered RNA polymerase II. Cell 18 613-622.
Gu Z, Nomura M, Simpson BB, Lei H, Feijen A, van den Eijnden-van Raaij J, Donahoe PK \& Li E 1998 The type I activin receptor ActRIB is required for egg cylinder organization and gastrulation in the mouse. Genes and Development 12 844-857.

Hu S \& Yang X 2003 Cellular inhibitor of apoptosis 1 and 2 are ubiquitin ligases for the apoptosis inducer Smac/DIABLO. Journal of Biological Chemistry 278 10055-10060.

Huppertz B, Kadyrov M \& Kingdom JC 2006 Apoptosis and its role in the trophoblast. American Journal of Obstetrics and Gynecology 195 29-39.

Hussein MR 2005 Apoptosis in the ovary: molecular mechanisms. Human Reproduction Update 11 162-177.

Hussein MR, Haemel AK \& Wood GS 2003 Apoptosis and melanoma: molecular mechanisms. Journal of Pathology 199 275-288.

Iannaccone PM, Zhou X, Khokha M, Boucher D \& Kuehn MR 1992 Insertional mutation of a gene involved in growth regulation of the early mouse embryo. Developmental Dynamics 194 198-208.

Jiang JY, Cheung CK, Wang Y \& Tsang BK 2003 Regulation of cell death and cell survival gene expression during ovarian follicular development and atresia. Frontiers in Bioscience 8 d222-d237.

Jones RL, Stoikos C, Findlay JK \& Salamonsen LA 2006 TGF-beta superfamily expression and actions in the endometrium and placenta. Reproduction 132 217-232.

Kim AH, Khursigara G, Sun X, Franke TF \& Chao MV 2001 Akt phosphorylates and negatively regulates apoptosis signal-regulating kinase 1. Molecular and Cellular Biology 21 893-901.

Kim BC, van Gelder H, Kim TA, Lee HJ, Baik KG, Chun HH, Lee DA, Choi KS \& Kim SJ 2004 Activin receptor-like kinase-7 induces apoptosis through activation of MAPKs in a Smad3-dependent mechanism in hepatoma cells. Journal of Biological Chemistry 279 28458-28465.

Kishimoto $H$, Nakagawa $K$, Watanabe T, Kitagawa D, Momose $H$, Seo J, Nishitai G, Shimizu N, Ohata S, Tanemura S et al. 2003 Different properties of SEK1 and MKK7 in dual phosphorylation of stress-induced activated protein kinase SAPK/JNK in embryonic stem cells. Journal of Biological Chemistry 278 16595-16601.

Labedzka K, Grzanka A \& Izdebska M 2006 Mitochondria and cell death. Postepy Higieny i Medycyny Doswiadczalnej 60 439-446.

Ma GT, Soloveva V, Tzeng SJ, Lowe LA, Pfendler KC, lannaccone PM, Kuehn MR \& Linzer DI 2001 Nodal regulates trophoblast differentiation and placental development. Developmental Biology 236 124-135.

Massague J 1998 TGF-beta signal transduction. Annual Review of Biochemistry 67 753-791.

Massague J \& Chen YG 2000 Controlling TGF-beta signaling. Genes and Development 14 627-644.

Miyazawa K, Shinozaki M, Hara T, Furuya T \& Miyazono K 2002 Two major Smad pathways in TGF-beta superfamily signalling. Genes to Cells 7 1191-1204.

Munir S, Xu G, Wu Y, Yang B, Lala PK \& Peng C 2004 Nodal and ALK7 inhibit proliferation and induce apoptosis in human trophoblast cells. Journal of Biological Chemistry 279 31277-31286.

Nakatani K, Sakaue H, Thompson DA, Weigel RJ \& Roth RA 1999 Identification of a human Akt3 (protein kinase B gamma) which contains the regulatory serine phosphorylation site. Biochemical and Biophysical Research Communications 257 906-910.

Peng C 2003 The TGF-beta superfamily and its roles in the human ovary and placenta. Journal of Obstetrics and Gynaecology Canada 25 834-844.

Reissmann E, Jornvall H, Blokzijl A, Andersson O, Chang C, Minchiotti G, Persico MG, Ibanez CF \& Brivanlou AH 2001 The orphan receptor ALK7 and the Activin receptor ALK4 mediate signaling by Nodal proteins during vertebrate development. Genes and Development 15 2010-2022.

Ries LA, Wingo PA, Miller DS, Howe HL, Weir HK, Rosenberg HM, Vernon SW, Cronin K \& Edwards BK 2000 The annual report to the nation on the status of cancer, 1973-1997, with a special section on colorectal cancer. Cancer 88 2398-2424.

Roberts HJ, Hu S, Qiu Q, Leung PC, Caniggia I, Gruslin A, Tsang B \& Peng C 2003 Identification of novel isoforms of activin receptor-like 
kinase 7 (ALK7) generated by alternative splicing and expression of ALK7 and its ligand, Nodal, in human placenta. Biology of Reproduction 68 1719-1726.

Schier AF \& Shen MM 2000 Nodal signalling in vertebrate development. Nature 403 385-389.

Suzuki Y, Nakabayashi Y \& Takahashi R 2001 Ubiquitin-protein ligase activity of $\mathrm{X}$-linked inhibitor of apoptosis protein promotes proteasomal degradation of caspase- 3 and enhances its antiapoptotic effect in Fas-induced cell death. PNAS 98 8662-8667.

Tsuchida K, Sawchenko PE, Nishikawa S \& Vale WW 1996 Molecular cloning of a novel type I receptor serine/threonine kinase for the TGF beta superfamily from rat brain. Molecular and Cellular Neurosciences 7 467-478.

Tsuchida K, Nakatani M, Yamakawa N, Hashimoto O, Hasegawa Y \& Sugino H 2004 Activin isoforms signal through type I receptor serine/threonine kinase ALK7. Molecular and Cellular Endocrinology 220 59-65.

Wang Y, Rippstein PU \& Tsang BK 2003 Role and gonadotrophic regulation of $\mathrm{X}$-linked inhibitor of apoptosis protein expression during rat ovarian follicular development in vitro. Biology of Reproduction 68 610-619.

Wang H, Jiang JY, Zhu C, Peng C \& Tsang BK 2006 Role and regulation of nodal/activin receptor-like kinase 7 signaling pathway in the control of ovarian follicular atresia. Molecular Endocrinology 20 2469-2482.

Xu G, Zhong Y, Munir S, Yang BB, Tsang BK \& Peng C 2004 Nodal induces apoptosis and inhibits proliferation in human epithelial ovarian cancer cells via activin receptor-like kinase 7. Journal of Clinical Endocrinology and Metabolism 89 5523-5534.
Xu G, Zhou H, Wang Q, Auersperg N \& Peng C 2006 Activin receptorlike kinase 7 induces apoptosis through up-regulation of Bax and down-regulation of Xiap in normal and malignant ovarian epithelial cell lines. Molecular Cancer Research 4 235-246.

Yang Y, Fang S, Jensen JP, Weissman AM \& Ashwell JD 2000 Ubiquitin protein ligase activity of IAPs and their degradation in proteasomes in response to apoptotic stimuli. Science $\mathbf{2 8 8} 874-877$.

Zhang L, Zhou H, Su Y, Sun Z, Zhang H, Zhang L, Zhang Y, Ning Y, Chen YG \& Meng A 2004 Zebrafish Dpr2 inhibits mesoderm induction by promoting degradation of nodal receptors. Science $\mathbf{3 0 6}$ 114-117.

Zhang N, Kumar M, Xu G, Ju W, Yoon T, Xu E, Huang X, Gaisano H, Peng C \& Wang Q 2006 Activin receptor-like kinase 7 induces apoptosis of pancreatic beta cells and beta cell lines. Diabetologia 49 506-518.

Zhou X, Sasaki H, Lowe L, Hogan BL \& Kuehn MR 1993 Nodal is a novel TGF-beta-like gene expressed in the mouse node during gastrulation. Nature 361 543-547.

Zinkel S, Gross A \& Yang E 2006 BCL2 family in DNA damage and cell cycle control. Cell Death and Differentiation 13 1351-1359.

Received 31 January 2007

First decision 22 February 2007

Accepted 22 March 2007 